\title{
Images et représentations des premières soldates françaises (1938-1962)
}

Images and Representations of the First French Female Soldiers (1938-1962)

\section{Élodie Jauneau}

\section{(2) OpenEdition \\ Journals}

Édition électronique

URL : http://journals.openedition.org/clio/9481

DOI : $10.4000 /$ clio.9481

ISSN : 1777-5299

Éditeur

Belin

Édition imprimée

Date de publication : 15 décembre 2009

Pagination : 231-252

ISSN : 1252-7017

\section{Référence électronique}

Élodie Jauneau, «Images et représentations des premières soldates françaises (1938-1962) », Clio.

Histoire, femmes et sociétés [En ligne], 30 | 2009, mis en ligne le 15 décembre 2012, consulté le 02 mai 2019. URL : http://journals.openedition.org/clio/9481 ; DOI : 10.4000/clio.9481 


\title{
Images et représentations des premières soldates françaises (1938-1962)
}

\author{
Élodie JAUNEAU
}

En 1938 est votée la loi «Paul-Boncour» sur l'organisation de la nation en temps de guerre. Pour la première fois, l'Armée française ouvre ses portes aux femmes, désormais mobilisables comme les hommes. En projet depuis 1927, cette loi donne lieu à de nombreux débats, alimentés par les théories antérieures - mais toujours actuelles - de la différenciation naturelle des rôles sexués.

L'un des obstacles majeurs à la féminisation des armées repose sur le concept de l'identité militaire comme une et indivisible, centrée sur le combat et l'usage de la force induisant la violence, le maniement des armes et le pouvoir d'ôter la vie, trois valeurs «naturellement» masculines observées et analysées dans les sociétés traditionnelles mais reproduites à l'envi dans les sociétés occidentales. L'organisation genrée de ces sociétés assigne la guerre, la chasse et le maniement des armes aux hommes et la sauvegarde du foyer et des valeurs familiales aux femmes. Paola Tabet démontre que c'est parce que le métier des armes reste un monopole masculin que les femmes se retrouvent exclues de la sphère de la chasse ou de la guerre. Ce qu'elle appelle le "sous-équipement» des femmes s'accentue et se confirme avec l'industrialisation des sociétés ${ }^{1}$. Alain Testard souligne que dans bien des sociétés, les femmes chassent comme les hommes, mais avec des armes différentes. Elles n'emploient jamais d'armes faisant couler le sang $^{2}$ mais ont recours à des armes «féminines » (chiens, gourdins, feu) pour une chasse «féminine». Parce qu'elles ne peuvent

\footnotetext{
1 Tabet $1979: 10$.

2 Sagaies, lances par exemple.
} 
provoquer l'écoulement du sang, l'accès aux armes destinées à trancher et ensanglanter leur est proscrit. Ce qu'il appelle « une affaire de sangs $»^{3}$ est en fait le fondement même de cet interdit qui semble relever d'une transgression anthropologique qu'auraient conservée nos sociétés ${ }^{4}$. Au début du $\mathrm{XX}^{\mathrm{e}}$ siècle, ce constat se vérifie toujours. Pourtant, si cette figure de $L A$ femme au feu reste marginale ${ }^{5}$, la Seconde Guerre mondiale, les guerres d'Indochine et d'Algérie voient augmenter les effectifs féminins en première ligne... bien que toujours désarmés, conformément aux lois militaires qui leur interdisent le port des armes et au poids de la représentation sexuée de la fonction guerrière. Les femmes ne peuvent, par nature, ôter la vie puisque leurs menstruations sont la garantie même de la pérennité de la nation - argument fréquemment repris dans les discours d'après-guerre, dans les sociétés occidentales contemporaines, quand l'heure est à la relance de la natalité. Pendant les deux guerres mondiales, où toutes les forces vives de la nation sont tournées vers l'économie de guerre, où les armes revêtent un caractère de destruction massive et où l'on parle de "guerre totale ", on imagine mal les femmes combattant aux côtés des hommes avec des armes différentes.

Ces arguments anthropologiques semblent justifier la sousreprésentation féminine dans l'armée ou l'inégalité persistante entre hommes et femmes pendant sa féminisation. Certains auteurs comme Martin Van Creveld considèrent que les femmes sont «nettement moins douées pour la guerre que les hommes » mais aussi plus faibles et vulnérables ${ }^{6}$. Soutenant que chacun-e a son propre rôle à jouer dans la société, les hommes doivent «protéger les femmes, plus faibles $[\ldots]$ en se battant pour elles.» Le rôle des femmes «ne comporte pas et ne doit pas comporter la guerre et les combats $»^{7}$. S'inscrivant contre la construction du genre, il pense aussi que le sexe - biologique - des chercheurs biaise leurs conclusions et leur

\footnotetext{
3 Testard $2005: 141$.

4 Audoin-Rouzeau $2005: 6$.

5 Seules les ambulancières et les infirmières sont officiellement en première ligne.

6 Van Creveld 2002: 18.

7 Van Creveld 2002 : 14.
} 
objectivité ${ }^{8}$. Enfin, il souligne que si la féminisation de l'armée s'est accélérée après 1970, c'est parce qu'elle est devenue une armée de dissuasion qui, en se féminisant, a contribué à son propre déclin.

Pourtant, il s'avère que les hommes ne sont pas naturellement des combattants. C'est leur sexe biologique qui engendre la construction sociale du guerrier. La vie de caserne doit construire la virilité et initier les hommes à leur fonction socialement « naturelle » de défense de la patrie. C'est ce que démontre Joshua S. Goldstein quand il affirme que la guerre ne vient pas biologiquement aux hommes mais que ce sont la socialisation et l'entraînement qui forgent le soldat. Ce sont les identités de genre traditionnelles qui conduisent la société à attribuer l'art de la guerre aux hommes ${ }^{10}$. A Martin Van Creveld qui souligne que les femmes n'étaient "pas mécontentes, à la fin du conflit, de quitter l'armée et de rentrer chez elles $»^{11}$, Joshua S. Goldstein répond que toute personne saine d'esprit et normalement constituée, homme ou femme, ne peut résister bien longtemps à la violence, la peur et la mort ${ }^{12}$. Les sources relatives au courage des femmes ou aux « faiblesses » de certains soldats prouvent alors que ce n'est pas la nature qui fait le guerrier, mais bien la société. Ainsi, loin de revendiquer la fonction masculine innée du combat, certains poilus de la Grande Guerre se souviennent de la crise identitaire engendrée par les atrocités du combat. Leur peur se manifeste parfois par des symptômes physiologiques et psychologiques traditionnellement attribués aux femmes tels que la somatisation, la folie, l'anxiété ou l'hystérie ${ }^{13}$.

8 Van Creveld $2002: 13-14$.

9 Van Creveld $2002: 297-298$.

10 Goldstein $2001: 252$.

11 Van Creveld $2002: 296$.

12 Goldstein 2001 : 253. "Any sane person, male or female, who is surrounded by the terrifying and surreal sights and sounds of the battle, instinctually wants to run away, or hunker down, and freeze up, and certainly not to charge into even greater danger to kill and maim other people ».

13 Rousseau 2003 : 181-190. 
Les seuls rôles féminins jusque-là tolérés sont donc ceux de soutien moral ou économique ${ }^{14}$, infirmières, prostituées, cantinières, vivandières ou activistes pacifistes ${ }^{15}$. Pourtant, dès 1939, les premières civiles sous l'autorité de l'armée, s'engagent dans des sections dépendant de la Croix Rouge. La fin de la « drôle de guerre » sonne le glas de ces sections mais pas celui de l'engagement féminin. À partir de 1940, elles sont plusieurs milliers à rejoindre les rangs des armées de libération de la France qui se mettent en place en Angleterre, en Afrique du Nord ${ }^{16}$ ou dans les Forces Françaises de l'Intérieur (FFI), intégrées à «l'Armée nouvelle » à la fin de la guerre. Lorsqu'éclate en 1945 la guerre d'Indochine, puis en 1954 celle d'Algérie, la mobilisation féminine est relancée avec le (ré)engagement de nombreuses volontaires.

Ces trois conflits s'accompagnent d'une augmentation constante des effectifs féminins dans l'Armée Française... mais non sans difficultés. En effet, entre 1938 et 1962, lois, décrets et ordonnances se multiplient pour «fixer» le statut de ces femmes et répondre à des questions qui ne s'étaient jusqu'alors jamais posées. Sur le plan culturel, cette «invasion » du féminin dans LE bastion masculin par excellence soulève des interrogations et suscite une curiosité grandissante pour ces militaires «d'un nouveau genre». Elles deviennent l'objet de fantasmes, d'études, rarement d'hommages, et focalisent de nombreux préjugés quant à la «nature » féminine, dénaturée par un engagement militaire "naturellement» masculin. Leur choix professionnel est vivement critiqué et souvent assimilé à une virilisation anormale. En choisissant cette voie, elles sont donc accusées d'aller à l'encontre des lois genrées de la société. L'explication puis l'analyse des représentations véhiculées par cet engagement militaire entendent démontrer que la féminisation de l'armée n'a pas débuté dans les années $1970^{17}$ mais qu'elle est bien

14 On pense alors par exemple aux marraines de guerre, aux munitionettes, ou aux femmes endossant les responsabilités économiques de leurs époux, frères ou pères, pendant la Première Guerre mondiale.

15 Goldstein 2001 : 251.

16 Après le débarquement allié de novembre 1942.

17 Problématique communément défendue par la plupart des travaux publiés sur cette question. 
née avec les guerres. Pour autant, ses conséquences ne furent pas celles attendues puisque les soldates restent encore aujourd'hui les oubliées de la mémoire combattante.

\section{Femmes et armées à la croisée des $\mathrm{XIX}^{\mathrm{e}}$ et $\mathrm{XX}^{\mathrm{e}}$ siècle}

L'image des femmes combattantes est souvent très péjorative. Même si elles sont l'objet de mythes ${ }^{18}$ ou de légendes, elles n'en sont pas pour autant glorifiées. Dans la Grèce classique, les amazones ${ }^{19}$ étaient le symbole d'un "monde à l'envers » et d'une inversion des rôles sexuels. Les plus célèbres sont celles du Dahomey ${ }^{20}$ au XIXe siècle, soldats féminins du roi. Devenu symbole de pouvoir féminin, ce terme est souvent utilisé par des femmes "pour légitimer leurs interventions dans les territoires masculins de la politique et de la guerre $»^{21}$.

$\mathrm{Au}$ moins jusqu'au XIXe siècle, la présence des femmes est remarquable au sein des troupes et non loin des champs de bataille. Elles sont cantinières, vivandières - souvent épouses ou veuves de soldats $^{22}$ ou encore prostituées. Mais depuis le décret du 30 avril 1793, l'armée obéit à une logique d'exclusion des femmes: la Convention, lasse des désordres occasionnés par leur présence, décide de les expulser, sauf les blanchisseuses et les vivandières indispensables au service des troupes. La dernière combattante du XIX $^{\mathrm{e}}$ siècle officiellement reconnue pour ses actes de bravoure est Marie-Angélique Brulon ${ }^{23}$, qui reçoit la Légion d'Honneur en 1851. La guerre de 1870 et la Première Guerre mondiale achèvent ce retrait des femmes de la sphère combattante. En 1914, la disparition de la

18 Steinberg 1999, cité dans Capdevila \& Godineau 2004 : 14. ; Cassagnes-Brouquet 2004 : 169-180 et $2003: 279-291$.

19 Évoquées pour la première fois dans l'œuvre d'Homère au VIII siècle avant JC : Zancarini-Fournel 2004 : 7-8.

20 Ancien nom du Bénin, dont le roi Agadja est à l'origine de ces régiments au XVIIIe siècle et dont l'effectif permanent était de 1600 femmes. Almeida-Topor 1984.

21 Zancarini-Fournel $2004: 8$.

22 Sorin $2002: 84$.

23 Déon-Bessière $2002: 35$ et Roynette $2002: 87$. 
figure de la cantinière en est l'illustration parfaite ${ }^{24}$. La seule présence féminine désormais tolérée est celle de l’infirmière, "personnage le plus louangé de la guerre ${ }^{25}$, parfois bénévole du service de santé militaire ${ }^{26}$.

Si l'armée cantonne les femmes à des rôles et des tâches compatibles avec leur sexe, c'est aussi par crainte de voir le vice de la «nature» féminine pénétrer la sphère militaire, pervertir les combattants et les détourner de leur mission guerrière, car les soldates sont vues comme des femmes viriles ou pernicieuses.

La conscription et le développement des casernes comme lieu initiatique de la virilisation du « citoyen-soldat $»^{27}$ excluent les femmes $\mathrm{du}$ monde militaire ${ }^{28}$. C'est depuis la fin de la Révolution que la citoyenneté masculine est devenue indissociable du service armé29. Et même si la figure de la femme semble indissociable de celle $d u$ soldat, sa militarisation est inenvisageable. La conscription accentue donc la frontière entre masculin et féminin dans la société : «la participation directe à la violence de guerre (donner ou recevoir la mort) dans un cadre étatique devient au début du $\mathrm{XX}^{\mathrm{e}}$ siècle, un critère qui distingue de manière plus nette que naguère hommes et femmes $»^{30}$. Les femmes liées à l'armée ou présentes dans les milieux traditionnellement masculins contribuent à une dénaturation de la femme. Cette virilisation de la «nature féminine» est fortement contestée car elle serait la cause d'un « amollissement $»^{31}$ des hommes. $\mathrm{Au}$ début du $\mathrm{XX}^{\mathrm{e}}$ siècle, seules les féministes radicales comme

24 Roynette $2002: 88$.

25 Thébaud $1986: 47-48$.

26 Sorin 2002 : 84. Les rares infirmières «salariées » du service de santé militaire sont en fait les premiers personnels féminins de l'histoire de l'armée. Elles sont entièrement « militarisées »: Ducret-Schaeffer $1980: 4$.

27 Zancarini-Fournel $2004: 10$.

28 «Virilisation» et «femmes» sont antinomiques car la virilisation est un processus modifiant la «nature» féminine en y intégrant des éléments traditionnellement masculins (comme le sport ou l'exercice de certains métiers), Zancarini-Fournel 2004 : 108-109.

29 Capdevila 2002 : 98.

30 Roynette $2002: 88$.

31 De la Barre Duparcq $1873: 304$. 
Madeleine Pelletier ${ }^{32}$, se posent en faveur d'un service féminin allant de pair avec l'égalité des droits et des devoirs ${ }^{33}$. Ce lien entre accès à la sphère militaire et à la citoyenneté politique est prégnant car Hubertine Auclert propose, elle aussi, un équivalent féminin au service militaire des hommes, soulignant que lorsque ceux-ci sont réformés, ils profitent au moins de leurs droits civiques ${ }^{34}$.

C'est dire si les débats sont déjà amorcés lorsqu'en 1927, un projet de loi envisage de mobiliser, en cas de guerre, toute la population sans distinction d'âge ni de sexe.

\section{La loi Paul-Boncour : objet des débats}

En 1927, Joseph Paul-Boncour, président du Conseil supérieur de la Défense nationale, est aussi rapporteur de la loi sur l'organisation de la nation en temps de guerre. Le projet envisage une mobilisation de toute la population en cas de conflit «sans distinction d'âge ni de sexe $\aleph^{35}$. C'est la première fois qu'une mobilisation des femmes est envisagée. 1927 marque le début de onze ans de débats, vivement critiqués par Joseph Paul-Boncour dans ses Souvenirs sur la III République ${ }^{36}$, car ils auraient considérablement retardé l'approbation de la loi et largement contribué à la défaite de la France en $1940^{37}$.

C'est la première fois qu'une telle mobilisation peut être contractée par une population qui jusqu'alors subissait la guerre sans y prendre part sur le plan juridique. Cette différence est extrêmement importante, notamment pour toutes les femmes qui ont contribué à l'effort de guerre pendant la Première Guerre mondiale et dont la «mobilisation » a été étouffée par le retour des héros en 1918.

En 1932, dans son roman Une vie nowvelle, Madeleine Pelletier imagine des femmes mobilisées et s'entraînant à la caserne :

Un projet déjà ancien sur la mobilisation générale de tout le pays avait été adopté. [...] Parmi les femmes, une élite vigoureuse était versée dans

\footnotetext{
32 Pelletier 1908.

33 Bard $1995: 141$.

34 Auclert $1908: 48$.

35 Loi du 11 juillet $1938: 8330$.

36 Paul-Boncour 1946.

37 Paul-Boncour $1946: 245$.
} 
le service armé. Depuis longtemps les femmes jouissaient de leurs droits politiques et on avait décidé qu'elles devaient comme les hommes l'impôt du sang. On découvrit alors que le service militaire n'exigeait pas tant de force qu'on le pensait et que la plupart des femmes de 20 ans bien portantes le pouvait faire sans dommage. [...] Pour le plus grand nombre, cette mobilisation ne changeait rien ${ }^{38}$.

$\mathrm{Si}$, pour les féministes, l'entre-deux-guerres est surtout marquée par la lutte pour l'accès à la citoyenneté politique, pour les radicales comme Madeleine Pelletier, elle s'accompagne aussi d'une égalité totale face à la lutte armée et la défense de la patrie ${ }^{39}$. A ses opposante-s qui la taxent d'excentricité et de bellicisme, Madeleine Pelletier répond que l'égalité absolue entre hommes et femmes passe aussi par l'impôt du sang. Elle est soutenue par Hélène Brion ${ }^{40}$ ou Arria Ly, toutes deux pourtant profondément pacifistes. D'autres, comme Marguerite Durand, souhaitent un service militaire auxiliaire pour les femmes. Elle le fait savoir le 4 mars 1927 dans le journal qu'elle dirige: La Fronde, tout comme Maria Vérone ${ }^{41}$ dans le Droit des Femmes, qui souligne cependant que la guerre, la mobilisation générale ou encore la diplomatie restent l'affaire des hommes et qu'une mobilisation féminine décrétée par eux relève d'une «injustice indigne de la démocratie $»^{42}$. Au contraire, certaines comme Cécile Brunschvicg refusent tout projet de mobilisation féminine. Toujours en 1927, Fernand Corcos, avocat favorable au féminisme, s'appuyant sur la complémentarité entre féminisme et pacifisme, soutient que la mobilisation des femmes n'augmenterait pas la force du pays mais contribuerait à accroître les pertes humaines et matérielles ${ }^{43}$. Enfin, il s'étonne du peu d'écho qu'a reçu ce débat dans la presse et de l'indifférence des féministes, tout en se demandant si ce manque d'intérêt vient du fait que personne ne croit en la mobilisation féminine ou si tout le monde croit que cela ne changera rien. Il serait

\footnotetext{
38 Pelletier 1932.

39 Pelletier 1908.

40 Bibliothèque Marguerite Durand : Fonds Hélène Brion.

41 Vérone : 97-99.

42 Vérone : 97.

43 Corcos 1927.
} 
très réducteur d'affirmer que les groupes féministes sont indifférents. Leur division semble être la raison majeure de leur silence.

Quoi qu'il en soit, l'argument le plus fréquent est celui de la pérennité de la nation et de la chute de la natalité qu'entraînerait une mobilisation des femmes. Pourtant, cette chute de la natalité engendrée par la mobilisation masculine n'est jamais évoquée tant elle semble « naturelle » au législateur. L'entre-deux-guerres, marquée par un excédent des décès sur les naissances, renvoie aux spectres de la dépopulation liée à chaque lendemain de guerre et déjà dénoncée par les anti-malthusiens après 1870. Cette faiblesse démographique française alimente la propagande populationniste et la politique familialiste faisant l'éloge de la femme au foyer ${ }^{44}$. Pour l'opinion dominante, le champ de bataille des femmes est celui de la maternité, la guerre celui des hommes. Comme l'a démontré Emmanuel Reynaud ${ }^{45}$, s'appuyant sur les travaux de Nancy L. Goldman ${ }^{46}$, «à l'image de l'homme biologiquement incapable d'enfanter, la femme serait par nature inapte à se battre : l'enfantement lui-même étant son propre combat et, finalement, son rôle militaire principal ».

Ce n'est que le 11 juillet 1938 que la loi est votée et dès lors, l'armée compte dans ses rangs les premières femmes servant sous statut civil. En 1939, la loi Paul-Boncour prend tout son sens et la mobilisation des femmes a pour but de «ne pas retomber dans les improvisations qui nous avaient coûté si cher en $1914 »^{47}$.

Pourtant, c'est l'Occupation, puis la création de la France Libre à Londres qui engendrent une nouvelle mobilisation féminine, dépassant largement les cadres définis par la loi. Pendant plus de quinze ans, les textes législatifs se multiplient pour tenter de donner un statut à ces femmes avec lesquelles les autorités militaires doivent désormais compter. L'engagement militaire féminin, bien que toléré mais souvent subi entre 1938 et 1962, permet désormais aux femmes un engagement militaire sur le long terme engendrant la mise en place d'une hiérarchie militaire ou encore la naissance d'un esprit de corps

\footnotetext{
44 Ripa $1999: 110$.

45 Reynaud $1988: 41$.

46 Goldman 1975 : 4-5, cité par Reynaud $1988: 41$.

47 Paul-Boncour $1946: 252$.
} 
féminin. Enfin, c'est l'augmentation constante des effectifs féminins entre ces deux dates qui est à l'origine des textes de loi et non l'inverse. C'est là toute l'originalité de ce processus qui met en évidence un écart considérable entre la théorie et le terrain. Ces militaires d'un "nouveau genre », parce que minoritaires, deviennent alors bien malgré elles des objets de questionnements ou d'enquêtes générant leur lot de préjugés, souvent plus proches du fantasme que de la réalité.

\section{Les pionnières de la Seconde Guerre mondiale}

Avant que la mobilisation féminine ne soit homogénéisée par la création des «formations féminines auxiliaires $»^{48}$ le 11 janvier 1944, les femmes s'engagent d'abord à Londres ou en Afrique du Nord. Les trois armes les accueillent et elles sont alors secrétaires d'État-major, transmissionistes, infirmières, ambulancières, sténodactylos, etc. Le 16 décembre 1941, est officiellement créé à Londres le Corps des Volontaires Françaises ${ }^{49}$. Finalement, cet enrôlement de la première heure est relativement bien accepté grâce aux termes d'un contrat d'engagement qui sont extrêmement clairs :

Le Corps des Volontaires Françaises constitue une formation militaire auxiliaire féminine, ayant pour objet :

- de libérer les combattants dont les emplois peuvent être tenus par des femmes.

- de doter les services militaires d'un personnel d'employés exclusivement militaire

Les volontaires s'engagent pour la durée de la guerre et trois mois après la cessation des hostilités ${ }^{50}$.

À contexte exceptionnel, engagements exceptionnels : préserver les valeurs morales de l'armée en acceptant les femmes à des postes conformes à leur nature et à leur sexe semble donc être une priorité. De plus, le retour à la paix annonce un retour «à la normale» semblable à celui de 1918. Pourtant, lorsqu'elles suivent leur

\footnotetext{
48 Décret du 11 janvier 1944 : 37.

49 Décret $n^{\circ} 74$ du 16 décembre 1941 : 3-4.

50 Décret $n^{\circ} 74$ du 16 décembre $1941: 3$.
} 
formation préliminaire, ces femmes foulent un terrain masculin ... qu'elles n'auront jamais l'occasion de retrouver! Ainsi, elles sont photographiées s'entraînant au tir ou s'exerçant au «parcours du combattant $»^{51}$, toujours en présence d'un supérieur masculin. Mais l'image de ces militaires de la première heure, bien qu'auxiliaires «à leur place », est loin d'être glorifiée. En effet :

On ne peut que constater l'incrédulité des spectateurs qui assistèrent aux défilés des femmes soldats ou des partisanes en armes à la Libération, et faire état de la mauvaise réputation dont souffrirent les «filles du maquis » et les engagées volontaires dans les FFL : l'engagement qu'elles avaient choisi dans la défense nationale et leur immersion dans un univers masculin n'étaient pas compris par le plus grand nombre ${ }^{52}$.

Les superstitions et les préjugés sont très vifs au sein de l'armée. Tout comme embarquer une femme sur un navire porte malheur, ce n'est pas sans crainte ni mise en garde que l'armée accepte les femmes. Celles qui s'engagent doivent être célibataires, veuves ou divorcées, sans enfant à charge, afin de ne pas troubler l'équilibre familial déjà mis à mal en contexte de guerre. Elles sont surveillées de près car les autorités craignent que "la femme mobilisée » ne soit «amenée à compromettre sa dignité ${ }^{53}$, à cause de la promiscuité avec les hommes ${ }^{54}$. Entre 1944-1945 et l'aube des années 1950, les rumeurs vont bon train concernant ces «femmes-soldats» qui sont souvent vues comme des «femmes au service des soldats ${ }^{55}$ ou comme des éléments perturbateurs pour l'armée mais aussi pour «ses hommes $\|^{56}$.

Toutes les femmes engagées dans l'armée pendant la Seconde Guerre mondiale subissent ce genre de critiques. Lorsqu'en 1943, Florence Conrad, une riche Américaine, à la tête d'une dizaine de femmes, envisage de rallier la $2^{\mathrm{e}}$ Division Blindée du général Leclerc,

51 Torrès 4 avril 2005 et Torrès : archives personnelles.

52 Capdevila \& Virgili 2000.

53 SHAT 7 P 73 : archives de la guerre. Vichy - Londres - Alger - Paris. 19401946 : organisation des formations féminines.

54 Le sigle «PFAT» a longtemps été employé de manière péjorative, et eu une connotation sexuelle : Sorin $2002: 87$.

55 Capdevila $2000: 72-74$.

56 Sorin $2002: 16$. 
celui-ci croit d'abord à " une blague »! Il n'est pas question d'enrôler des femmes dans une division blindée. L'engagement des femmes dans l'armée et les différents appels que la France Libre a lancés sont clairs : les femmes qui s'engagent le font pour libérer un combattant, non pour combattre elles-mêmes. En effet, la France Libre a accéléré en 1943 sa campagne en faveur du recrutement féminin appelant les Françaises à s'engager afin de permettre à tout homme, capable de combattre, d'être libéré des tâches qu'une femme peut accomplir, ce qui n'inclut évidemment pas la sphère du combat. Finalement, Leclerc les accepte avec réticence en précisant qu'elles ne poursuivront pas au-delà de Paris ${ }^{57}$. Ces femmes sont rapidement surnommées "Rochambelles» par les hommes de la 2e DB. Mais l'origine même de ce surnom interpelle. Baptisé par elles «Groupe Rochambeau », en hommage au compagnon de Lafayette lors de la guerre d'indépendance américaine, celui-ci peut alors paraitre à première vue très connoté sexuellement... mais aussi militairement. Tous les «groupes» de femmes militaires sont féminisés ou comportent le mot «féminin-e » dans leur nom. Les transmissionistes sont les «Merlinettes $»^{58}$ et les «Marinettes» sont les premières femmes engagées dans la Marine de la France Libre ${ }^{59}$. Les recrues de l'Armée de l'Air deviennent rapidement les «filles de l'air»... Le Groupe Rochambeau, bien que rapidement féminisé par les hommes de la $2^{\text {e }} \mathrm{DB}$ demeure aujourd'hui le seul groupe féminin de l'armée dont le nom « officiel» est resté masculin. Ainsi, les archives relatives aux Rochambelles sont conservées en tant que "Groupe Rochambeau $»^{60}$. Sa féminisation par les hommes de la 2e DB invite donc à réfléchir sur ce besoin de distinguer hommes et femmes dans l'armée. L'hypothèse selon laquelle elle intervient dans un souci de distinction et de non-assimilation des femmes se pose.

57 Il se ravisera finalement face au courage dont elles auront fait preuve et iront bien au-delà de Paris puisque nombre d'entre elles suivront Leclerc jusqu'en Indochine.

58 Fondateur du Corps Féminin des Transmissions (CFT) en 1943.

59 Affectées au régiment blindé de fusiliers marins (RBFM) de la 2e DB.

60 Fonds du Mémorial du maréchal Leclerc. 
Enfin, l'armée ne féminise jamais les grades, et les expressions « commandante » ou « lieutenante » par exemple restent officieuses et exclusivement réservées aux femmes entre elles. Employées par les hommes, elles sont péjoratives ou ironiques ${ }^{61}$. Les archives sur ce sujet sont très révélatrices : les grades sont toujours masculins et les femmes toujours désignées en tant que telles ${ }^{62}$. Mais parfois, des notes de service ou des courriers laissent apparaittre une réelle difficulté qu'ont les hommes à identifier ces femmes. En témoigne une note de service du 6 novembre 1942, dans laquelle il est question des « sous-officiers et hommes de troupe féminins $»^{63}$.

Les obstacles auxquels ces "pionnières" se heurtent, persistent après la guerre, à l'heure des décorations et de la reconnaissance pour «services rendus à la Nation ». Elles sont alors très peu représentées parmi les Compagnons de la Libération ${ }^{64}$ ou encore très tardivement décorées de la Légion d'Honneur, telles les Rochambelles qui l'obtiennent plus de trente ans après les faits ${ }^{65}$.

Lorsqu'immédiatement après la fin du second conflit mondial, éclate la guerre d'Indochine, bon nombre de femmes «rempilent». Ce contexte particulier est propice à un intérêt grandissant - qui ne sert pas toujours la cause de ces militaires de l'autre sexe.

\section{Un intérêt nouveau pour ces militaires d'un « nouveau genre »}

$\mathrm{Si}$ quelques hommages au sein de l'armée prouvent une certaine reconnaissance, ceux-ci restent assez limités et les réflexions nouvelles sur ces femmes aboutissent plutôt à des conclusions mitigées et parfois très critiques. En dépit de leurs exploits ou de leur

61 Jauneau 2008.

62 AFAT, et puis PFAT (Personnel Féminin de l'Armée de Terre), Formations Féminines de l'Armée de l'Air (FFAA), Sections Féminines de la Flotte (SFF) et plus tard Personnel Féminin de la Marine (PFM), Infirmières Parachutistes Secouristes de l'Air (IPSA). Ce n'est que dans les années 1960 que l'acronyme PMF pour Personnels Militaires Féminins tend à se généraliser.

63 SHAT : 4 H 337-7: note de service 16092/3 du 6 novembre 1942 de la délégation de la France Libre au Levant.

64 Capdevila \& Virgili 2000.

65 Jauneau, à paraître. 
dévouement, l'idée alors la plus répandue est que la place des femmes, même en temps de guerre, est au foyer :

Les femmes comme les hommes, mais à leur place, ont à se mettre au service du pays. La participation à l'effort de guerre entraîne des bouleversements profonds mettant en jeu la dignité et la mission de la femme, le moral des soldats, la conduite de la guerre et l'Avenir du Pays. [...] Même en temps de guerre, le rôle primordial de la femme est au foyer ${ }^{66}$.

En bravant les lois du genre, ces femmes s'exposent au regard de la société, des médecins et des politiques, et le spectre des discours essentialistes et naturalistes revient parfois hanter leurs conclusions. Lorsqu'Hélène Taillefer soutient sa thèse de Médecine en 1947, elle se pose en faveur d'un contrôle psychique sévère à l'engagement des femmes. Loin des discours classiques, elle démontre pourtant que les femmes ont prouvé leurs capacités à s'engager dans les guerres et elle soutient qu'aucune considération psychologique ou physiologique ne s'oppose à leur incorporation dans l'armée. Toutefois, elle met en garde le recruteur :

Aujourd'hui que la guerre est finie, beaucoup estiment que l'armée féminine ne sert plus à rien, cependant on peut dire, sans être taxée d'exagération, que dans un pays comme le nôtre, où le chiffre de la population est relativement faible par rapport à nos charges politiques et militaires, il sera nécessaire, chaque fois que nous nous trouverons en état de guerre, de faire appel aux femmes.

[...] Si parmi les héroïnes des temps anciens, beaucoup étaient animées de sentiments éthiques, il est incontestable que d'autres peuvent apparaître, même à travers l'histoire, comme des déséquilibrées.

[...] Il est incontestable que l'armée féminine, composée d'engagées, doit éliminer les psychopathes avec plus de rigueur encore que dans l'armée masculine, armée de conscription ${ }^{67}$.

66 SHAT, 7 P 73-1: Gilda Sinon, déléguée des mouvements féminins, Jean Fontensau, président du Conseil auprès du chef de la Jeunesse et Jean de Chelle, chef du service de la Jeunesse et des Sports au Commissariat à l'Intérieur. Sans date mais classé dans un dossier intitulé : «Le service féminin aux Armées et l'avenir de la France - 1945 ».

67 Taillefer $1947: 35$. 
Ses arguments amènent deux remarques. Tout d'abord, elle semble oublier que la France est toujours en guerre à l'heure où elle écrit mais surtout elle laisse entendre que les femmes ne sont plus mobilisées. S'il est vrai qu'elles sont nombreuses à quitter l'armée quelques mois après la fin des hostilités, elles sont plusieurs milliers à signer un nouvel engagement pour l'Extrême-Orient. Enfin, elle souligne l'inégalité du contrôle psychique entre hommes et femmes lors de leur engagement. Or, l'article 3 du décret $n^{\circ} 74$ du 16 juin 1941 stipule pourtant que «l'admission est subordonnée au résultat favorable d'une enquête de moralité [et l'] aptitude physique est constatée par un examen médical d'incorporation $»^{68}$. Le décret du 11 janvier 1944 ajoute que «sont exclues des formations militaires féminines auxiliaires les femmes se livrant à la prostitution, les femmes ayant fait l'objet d'une condamnation privative d'au moins quinze jours, inscrite au casier judiciaire $»^{69}$. La psychopathie se manifestant par un «trouble permanent de la personnalité, n'étant ni de l'ordre de la psychose ni de la névrose et se caractérisant essentiellement par des conduites antisociales impulsives dont le sujet ne ressent habituellement pas de culpabilité $»^{70}$, on imagine mal comment de tels individus peuvent échapper aux autorités. Quoi qu'il en soit, le regard de l'historien-ne doit être prudent puisque la pratique de la psychiatrie dans l'armée n'existe que depuis le début du $\mathrm{XX}^{\mathrm{e}}$ siècle. Cela vient du fait que le milieu militaire semble exercer une influence incontestable sur l'éclosion et le développement des maladies mentales ${ }^{71}$. La liste des psychopathologies ${ }^{72}$ dans l'armée est la même pour les hommes et les femmes. Pourtant, la place que l'auteure accorde à certains déséquilibres, tels que l'homosexualité, est considérablement disproportionnée selon qu'elle traite des militaires masculins ou féminins. Ainsi, les «attentats aux mœurs » des hommes sont tout juste signalés tandis que les «perversions sexuelles»

\footnotetext{
68 Décret $n^{\circ} 74$ du 16 décembre $1941: 3$.

69 Décret du 11 janvier $1944: 38$.

70 Postel $1993: 520$.

71 Taillefer $1947: 39$.

72 Débiles mentaux, déséquilibrés, états dépressifs, hystérie, épilepsie, cyclothymie, démence précoce, paranoïa, confusion mentale, toxicomanies, etc.
} 
féminines occupent une place de choix dans le chapitre consacré aux «déséquilibres pervers ». Le mythe selon lequel l'armée féminine concentrerait une plus forte proportion de lesbiennes que dans le reste de la société est très répandu. Il obéit à des théories déjà anciennes du complexe de virilité 73 des femmes souffrant de troubles identitaires. Celles-ci choisiraient alors une voie traditionnellement masculine et contre-nature pour vivre plus facilement leur homosexualité dans un cantonnement militaire non-mixte :

Il existe deux sortes de lesbiennes: les lesbiennes actives qui prennent des manières masculines tant dans leur comportement extérieur que dans leur pensée. Ce sont des femmes d'allures masculines dans leur habillement et dans leur façon de parler qui font du prosélytisme et qui, par là, sont les plus dangereuses.

Les lesbiennes passives de caractère souvent faible, qui recherchent «à moindre frais» dans les mœurs de Lesbos une forme d'érotisme symbolique.

Ce problème n'a pas tardé à préoccuper le commandement féminin

[...] Le cantonnement des AFAT, à Paris en particulier, a dû être improvisé, on fut même obligé de les coucher par deux. [...] Parmi les faibles, beaucoup devaient devenir une proie et une victime facile au prosélytisme de certaines perverses [...] Il est donc indispensable de savoir reconnaître les lesbiennes perverses qui doivent être éliminées de façon absolue ${ }^{74}$.

Si rien ne prouve une telle inquiétude du commandement féminin, la question mérite d'être posée. Tereska Torrès, Volontaire Féminine de la première heure, est beaucoup moins catégorique qu'Hélène Taillefer lorsqu'elle évoque l'homosexualité féminine dans l'armée. Si elle reconnaît volontiers la présence de quelques lesbiennes dans sa caserne $^{75}$, elle affirme également qu'aucune n'a «jamais essayé de séduire celles qui ne l'étaient pas [...] et cela ne devait pas être jugé si épouvantable que ça puisqu'on a nommé l'une d'elles adjudante $»^{76}$. L'histoire des homosexualités dans l'Armée française reste à faire,

\footnotetext{
73 Deutsch 1949 : 240-276.

74 Taillefer $1947: 92$.

75 Jauneau 2008.

76 Torrès 4 avril 2005.
} 
contrairement aux études anglophones déjà nombreuses ${ }^{77}$. En 1953, une autre thèse de Médecine est soutenue sur le même thème. Jacques Sarraz-Bonnet se défend en affirmant qu'il n'est pas prématuré de retravailler cette question puisque les travaux d'Hélène Taillefer portent :

sur les premiers contingents admis dans l'armée comme auxiliaires, et dont l'organisation nouvelle pouvait laisser prévoir un certain nombre de critiques. D'une part, et ceci est essentiel, ces premiers contingents ont été constitués dans une période de guerre, donc dans une période où la qualité devait nécessairement être sacrifiée à la quantité. D’autre part, le recrutement s'est effectué dans la période d'excitation de la Libération $»^{78}$.

Il exclut donc de l'étude d'Hélène Taillefer toutes celles qui étaient déjà dans l'armée avant que ne soit constituée l'AFAT, mais il considère clairement que la qualité de cette armée féminine laissait plus qu’à désirer.

Dans les années 1950, la question de la masculinisation voire l'assexuation des femmes militaires n'est pas soulevée que par les médecins. Pierre Carles, colonel ${ }^{79}$ présent en Indochine, se souvient que les hommes se retenaient dans leurs propos les plus crus en présence des femmes dans les mess. Mais petit à petit, les «mauvaises habitudes» reprennent le dessus et ce sont les femmes qui ont du :

habituer leurs oreilles à entendre des conversations plus ou moins gaillardes et parfois même grossières. [...] Il semble que ce ne soient pas les femmes qui aient poli les hommes mais bien les hommes qui aient amené la création d'un type militaire féminin. [...] Vers 1955, après l'Indochine, après dix ans de services féminins, on commence à voir quelques rudes gaillardes, souvent décorées, et dont la féminité ne s'étend pas toujours au langage. [...] Célibataires ou " démariées ", ces adjudantes créent, hélas, un pendant féminin aux vieux sous-officiers gueulards qui ont fait la fortune des opérettes militaires. Compétentes le plus souvent, elles finissent par se rassembler dans les unités

77 Berube 1990.

78 Sarraz-Bonnet $1953: 16$.

79 Ancien président du Centre d'histoire militaire et d'études de défense nationale de Montpellier. 
d'instruction des personnels féminins, où elles font les débuts difficiles aux jeunes engagées ${ }^{80}$.

Il serait évidemment déraisonnable de généraliser sur les tendances masculines ou homosexuelles des soldates mais les sources permettent d'affirmer qu'elles ont suscité un regain d'intérêt et relancé les débats sur la nature féminine. Enfin, l'image qu'ont les militaires masculins de ces femmes est tout aussi inégale. Si certains comme Pierre Carles, Roger Delpey ${ }^{81}$ ou Jacques Massu ont plutôt tendance à leur rendre hommage, d'autres comme le quartier-maittre Wattel ${ }^{82}$ en 1957 leur sont clairement opposés :

Jamais la femme ne sera l'égale de l'homme dans ce métier [...] Si ces femmes sont souvent considérées avec « dédain » ou « condescendance », c'est souvent, il faut le dire, avec juste raison [...] La femme-soldat est un être hybride et asexué bien loin de l'ange au foyer [...] Le plus beau rôle de la femme n'est-il pas celui d'être épouse et mère [...] Restez à votre place et laissez le métier des armes à vos fils et à vos maris ${ }^{83}$.

Pendant ces décennies de guerre continue, l'image des femmes soldats est assez malmenée. La difficulté qu'éprouve la culture collective à les assimiler repose surtout sur la persistance des rôles sexués traditionnels. C'est en effet en contexte de conflit que les rapports sociaux de sexe semblent alors le plus mis à mal et que les rôles dits «naturellement sexués» s'ébranlent. Néanmoins, la mémoire collective peine à intégrer ces personnels militaires de l'autre sexe tant dans les commémorations, que sur la pierre des monuments aux morts ou dans les livres d'histoire. Même si l'enseignement de l'engagement féminin pendant la Seconde Guerre mondiale se met

80 Carles 1982 (1959) : 104.

81 Journaliste et écrivain, il est mobilisé en Indochine avec le grade de sergent et également correspondant de guerre.

82 Grade de la Marine nationale équivalent à caporal (quartier-maittre 2e classe) ou caporal-chef (quartier-maitre $1^{\text {re }}$ classe).

83 Q.M. Wattel, Cherbourg, "Réponse à Yvette», Bellone, 43, juillet-août 1957, p. 30. A noter que dans la Marine, plus que dans les autres armes, les femmes ont du faire face à davantage de préjugés dont le plus célèbre est celui qui veut qu'embarquer une femme porte malheur. 
progressivement en place, la présence des femmes en Indochine et en Algérie demeure occultée. L'idée selon laquelle, parce que peu nombreuses, les soldates auraient été l'objet d'hommages appuyés ou d'héroïsation particulière est donc fausse. Parce que celle selon laquelle la féminisation de l'armée aurait débuté dans les années 1970 est communément admise, la trace des femmes qui se sont engagées avant est perdue. La figure du héros étant masculine dans l'imaginaire collectif, la place accordée aux femmes «mortes pour la France», «tombées au champ d'honneur» ou honorées pour «services rendus à la nation » est donc inexistante. En endossant l'uniforme du soldat, elles ont du affronter les barrières culturelles les plus solides. Elles ont subi une mise à l'écart de la mémoire combattante et donc de la mémoire collective. Rendre aux femmes soldats la place qui est la leur dans l'historiographie militaire française reste à faire. Quant aux guerres d'Algérie et d'Indochine, bien qu'elles se soient conclues par des défaites, les monuments aux morts ne manquent jamais de mentionner les hommes qui sont tombés pour la France. La victoire n'apparait donc pas non plus comme un facteur d'héroïsation ou de reconnaissance. La seule explication valable pour justifier l'absence des femmes semble donc être celle de leur sexe. En 2004, Solange Cuvillier-Simon ${ }^{84}$ publie une Lettre dans laquelle elle déplore le peu de cas qu'il est fait des femmes vétérans :

Cette lettre ouverte résulte du sentiment d'abandon ressenti depuis soixante ans par des femmes vétérans, premières engagées volontaires $[\ldots]$ avec, dans le cœur l'amour de la Patrie et la volonté de la libérer du joug allemand. [...] 15 août 2004 - Soixantenaire du débarquement de Provence : Pour nous, femmes vétérans d'Italie, cet anniversaire aura été notre plus grande déception. La troisième chaîne officie dans sa bulle à 100 mètres de nous. Mais nous restons isolées et ignorées ${ }^{85}$.

Elle conclut sa lettre par le regret qu'elle a de voir la campagne d'Italie absente des livres d'histoire: «Les élèves sont en droit d'apprendre les actes de bravoure de leurs pères, grands-pères... et arrières grands-pères... et grands-mères !! » Nombreuses sont celles,

\footnotetext{
84 Ambulancière au sein du Corps Expéditionnaire Français en Italie dès 1943.

85 Cuvillier-Simon 2004.
} 
comme Solange Cuvillier-Simon ${ }^{86}$, qui ont choisi de se raconter, soit immédiatement après les faits, soit bien des années plus tard. En laissant une trace écrite de leur(s) guerre(s), elles posent la première pierre d'une transmission féminine de la mémoire combattante.

\section{Bibliographie}

Audoin-RouZEAu Stéphane, 20 janvier 2005, « La violence de guerre au XXe siècle : un regard d'anthropologie historique ", Les rendez-vous du CHEAr-Ministère de la Défense - DGA, disponible sur internet.

BARD Christine, 1995, Les filles de Marianne. Histoire des féminismes 1914-1940, Paris, Fayard.

BERUBE Alan, 1990, Coming out under fire. The history of gay men and women in the World War II, New York, Plume.

CAPDEVILA Luc, 2000, "La mobilisation des femmes dans la France combattante (1940-1945) », Clio, Histoire, Femmes et Sociétés, 12, p. 57-80.

—, 2002, "L'identité masculine et les fatigues de la guerre », Vingtième siècle. Revue d'histoire, 75, p. 97-108.

Capdevila Luc \& Dominique Godineau (dir.), 2004, «Armées», Clio Histoire Femmes et Sociétés, 20.

Capdevila luc, Cassagnes Sophie, Cocaud Martine, Godineau Dominique, RouQUeT François \& Jacqueline SAINCLIVIER (dir.), 2003, Le genre face aux mutations. Masculin, Féminin, du Moyen Age à nos jours, Paris, Presses Universitaires de Rennes.

CapdeVila Luc \& Fabrice Virgili, 2000, Guerre, femmes et nation en France (19391945), disponible sur internet.

CARLes Pierre, 1982 (1 19e éd. 1959), Des millions de soldats inconnus, La vie de tous les jours dans les armées de la IVe République, Paris, Lavauzelle.

CoRCos Fernand, 1927, Les femmes en guerre, Paris, Montaigne.

CuviluIER Solange, 1991, Tribulations d'une femme dans l'Armée française, Ou le patriotisme écorché, Paris, Lettres du Monde.

—, 2004, Lettre ouverte du 2 septembre 2004 à Messieurs les directeurs des principaux quotidiens français, disponible sur internet.

86 Cuvillier-Simon 1991. 
DelPey Roger, 1969, La bataille du Tonkin, Soldats de la boue, tome 2, Paris, J'ai lu (coll. « Documents »).

DÉON-BESSIÈrE Danièle, 2002, Les femmes et la Légion d'Honneur depuis sa création, Paris, Éditions de l'Officine.

Deutsch Hélène, 1949, Psychologie des femmes. Étude psychanalytique, Paris, PUF.

Ducret-SchaEFFer Odile, 1980, Les femmes dans les armées en France : 1914-1979, mémoire de Sciences Politiques, Paris, Fondation nationale des sciences politiques, Centre de Sociologie de la Défense nationale.

Goldstein Joshua S., 2001, W ar and Gender, Cambridge, University Press.

JAUNEAU Elodie, 2008, «Des femmes dans la France combattante pendant la Deuxième Guerre Mondiale : le Corps des Volontaires Françaises et le Groupe Rochambeau », Genre \& Histoire, 3, disponible sur internet.

LA BARre DduparcQ Edouard de, 1873, Histoire militaire des femmes, Paris, aux frais de l'auteur.

Paul-Boncour Joseph, 1945-1946, Souvenirs sur la III République, Entre deux guerres, Paris, Plon.

Pelletier Madeleine, octobre 1908, «La femme soldat », La Suffragiste.

—, 1932, Une vie nouvelle, Paris, E. Figuière.

Postel Jacques, 1993, Dictionnaire de psychiatrie et de psychopathologie clinique, Paris, Larousse.

REYNAUD Emmanuel, 1988, Les femmes, la violence et l'armée, essai sur la féminisation des armées, Paris, FEDN.

Rousseau Frédéric, 2003 (1 re éd. 1999), La guerre censurée, Paris, Seuil.

ROYNETTE Odile, 2002, «La construction du masculin de la fin du XIX ${ }^{\mathrm{e}}$ siècle aux années 1930 », Vingtième Siècle. Revue d'histoire, 75, p. 85-96.

SARraz-Bonnet Jacques, 1953, Contribution à l'étude de la psychopathologie dans le personnel féminin de l'armée, Paris, Imprimerie R. Foulon.

SORIN Katia, 2002, Femmes en armes, une place introuvable? Le cas de la féminisation de l'Armée française, Thèse de doctorat en Sociologie sous la direction de François Gresle, Université de Paris I, publiée sous le même titre en 2003 aux éditions L'Harmattan.

TABET Paola, 1979, «Les mains, les outils, les armes », L'Homme, xIX, 3-4 p. 5-62.

Testard Alain, 2005, "La femme et la chasse », in Françoise Héritier (dir.), Hommes, femmes la construction de la différence, Paris, Le Pommier / Cité des Sciences et de l'Industrie, p. 137-150. 
TAILlEFer Yvonne-Hélène, 1947, Essai de psycho-pathologie féminine dans l'armée, Paris, Imprimerie Maurice Lavergne.

THÉBAud Françoise, 1986, La femme au temps de la guerre de 14, Paris, Stock.

VAN CREVELD Martin, 2002, Les femmes et la guerre, Monaco, Le Rocher.

ZANCARINI-Fournel Michelle, 2004, Les mots de l'Histoire des femmes, Toulouse, Presses Universitaires du Mirail. 